Chapter 9, Ocular Surface Immune Reactions and Contact Lens Wear, has only two references from the 1990s and the more recent of these is 1993 . It contains nothing that is not common knowledge amongst contact lens practitioners.

Chapter 10, Keratitis and Contact Lenses, is a brief but useful overview of the present thoughts and state of the art in the field of disposable lenses. Its critical and economical style makes it a pleasure to read. I am suspicious that Figure 10.2 is of a corneal nerve and not Aspergillus fumigatus. The chapter ends with the not surprising conclusion that daily-disposable contact lenses when worn as prescribed are safest.

I was left wondering why this book was assembled, what it was intended to do and for what audience.

P.N. Dilly

\section{The Eye in General Practice}

R.D. Finlay and P.A.G. Payne Heinemann, 1997

An interesting little book this, with much to commend it to the doctor who wishes to have a quick and comprehensive overview of common ocular problems, particularly if there is little desire to know what the specialist might do to treat a particular condition. I think it might be an excellent primer for a student or a very good basic background text for a GP registrar. Unfortunately I do not think it begins in any way to live up to its title. I think more appropriately termed 'Ophthalmic Problems for the Uninitiated' it would do very well.

Sadly the book starts badly, suggesting as it does that an essential tool for the examination of the eye in general practice is a slit lamp. I performed a straw poll of a number of GP colleagues and was able to ascertain that, although many actually knew that a slit lamp was a tool used by an ophthalmologist, none could with any certainty identify where they would expect to find the on/off switch. Not surprisingly I was also unable to find a GP who owned a slit lamp. We really can do without books for general practice which clearly demonstrate such a lack of understanding of the role of the GP in managing ophthalmic conditions.

General practitioners, on the other hand, have a very different agenda in their management of eye disorders in their patients. Put simply they require to know what they may safely diagnose and treat and what they must refer and what they must not attempt to treat. All the information required to perform these functions is, I am sure, available within the pages of this book, it is just that, organised as it is, the material is inaccessible to the GP rapidly.

An example of this is the series of tables which appears in Chapter 2. Table 2.1 is titled 'The Acute Red Eye' and contains a series of causes of the acute red eye with a clinical description

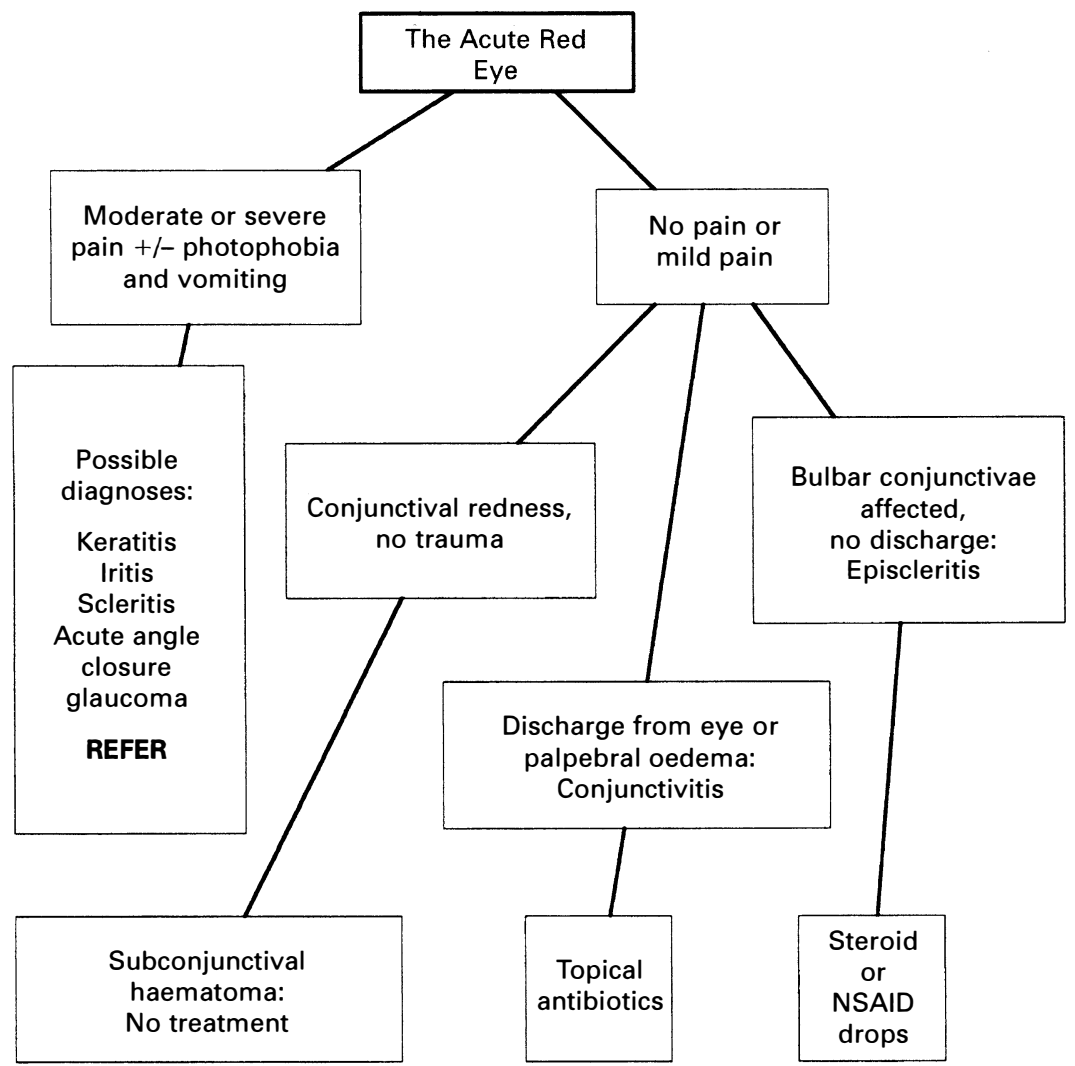

Fig. 1. followed by a treatment. Four of the conditions described have a suggested treatment of 'refer'. Surely a more appropriate vade-mecum of GP ophthalmological problems would use a decision tree such as that in Fig. 1 to inform the GP of appropriate diagnosis and/or disposal.

Thus this very good little book could be excellent if its content were reorganised to be useful to its target audience. I think that a small primer which gave a sufficiently easy to use series of diagnostic algorithms would rapidly gain acceptance as a GP standard text - a position which I fear this book is not destined to occupy. John Poyser

Ophthalmology - Colour Guide,
2nd edition

Jack J. Kanski

Churchill Livingstone, 1997

\section{Pocket Book of Ophthalmology}

Philip I. Murray and Alistair R. Fielder Butterworth-Heinemann, 1997

'. . . vether it's worth while goin' through so much to learn so little, as the charity boy said ven he got to the end of the alphabet, is a matter of taste', said Mr Weller in Pickwick Papers. For those without the time or money to invest in the third edition of Clinical Ophthalmology, JJK's Colour Guide is the 'charity boy' edition.

It would be a breach of hippocratic dogma for any British-trained ophthalmologist of my generation to have anything negative to say about any book by JJK. This volume is a pocketsized and slimline condensation of the great tome on which we were all raised. It contains many of the same beautiful illustrations with a pithy bullet-form commentary accompanying. The quality of the illustrations is uniformly excellent (e.g. Vogt's striae on p. 38). It will, no doubt, be popular among medical students and brand-new SHOs, for whom the visual impact and verbal brevity will be very appealing, but it has little to offer anyone who will have to invest in the larger volume for their future training.

Aimed at much the same audience, Murray and Fielder's book is the same size and has not one photograph in it, which will make it hard to sell. It is a book of lists, despite the disclaimer in the introduction. It is likely to be perceived as too specialist by the generalist (who wants to see pictures) and inadequate by the specialist trainee (who will have invested in a well-known and more encyclopaedic book of ophthalmic lists - also without pictures). It is not consistent in the level and pitch of the script, in which it is felt necessary to explain the word 'stye' ('infection of eye lash root') but leaves 'oscillopsia' 
undefined on the next page. Having sat at the didactic feet of both authors it is with regret that I find little to enthuse about in this volume.

Neil Rogers

\section{Clinical Procedures: Lids and Nasolacrimal System}

Eric E. Schmidt

Butterworth-Heinemann, 1997

ISBN 0-7506-9621-4

This book is from a series of practical guides aimed at optometrists in North America. It is divided into five chapters: dry eye, wet eye, eyelash disorders, benign lid lesions and finally infectious and inflammatory eyelid disease. Each chapter begins with a brief outline of relevant anatomy and physiology; clinical features and useful diagnostic procedures are described in detail, followed by treatment options, again with a full description of therapeutic procedures including local anaesthesia. The end of each chapter covers billing and reimbursement as well as North American insurance codes. This pocket guide has much to offer in the often groaned about area of eyelid disorders and with it we can get away from the 'see one, do one, teach one' philosophy. It has lots of drawn illustrations which will be useful not only to trainee ophthalmologists but also to the everevolving ophthalmic nurse practitioner as well as optometrists in the UK, particularly as their role in primary eye care is likely to expand in the future.

Visual Fields: Examination and Irene Whelehan

Interpretation. Ophthalmology Monograph 3, 2nd edition

Thomas J. Walsh

American Academy of Ophthalmology, 1997
This monograph is written by Thomas Walsh with special contributions from four colleagues who have expertise in neuro-ophthalmology and glaucoma. The book is aimed at the resident in ophthalmology and practising clinicians. The emphasis upon practical advice is entirely appropriate; ophthalmologists should fully understand and participate in field testing both to appreciate the tests their patients undergo and to make valid management decisions. There are plenty of useful tips, the illustrations are clear and helpful and there are adequate references.

The first two chapters introduce the techniques of field testing and give an overview of the visual pathway. The section on the anatomy of the striate cortex is especially fascinating. Advances made from functional magnetic resonance studies and computer graphics have greatly expanded the proportional area of macular projection, demonstrated a remarkable variability in the orientation of the calcarine fissure in different human brains and mapped the extrastriate visual cortex.

There are two chapters on automated perimetry and its application to the management of glaucoma. There is a good exposition of the principles of Humphrey automated perimetry and the information that may be gleaned from the printout and statistical programmes. The evaluation of a series of automated field tests provides a concise guide to the difficulties produced by artefacts, short- and longterm fluctuations, the learning effect and the criteria for diagnosing abnormality and progression of glaucomatous field defects. Throughout it is emphasised that published schemes are only guidelines to be considered in conjunction with many other clinical factors in guiding treatment.
The remaining chapters describe the field defects found with specific abnormalities of the visual pathway from the retina to the occipital cortex. A full range of neuro-ophthalmological disease is illustrated. Controversies are aired, and the commentary is not dogmatic. Wilbrand's knee is no longer perpetuated since its existence has been undermined by Jonathan Horton's anatomical studies. The tests for motor impersistence and the extinction phenomenon in patients with parietal lobe deficits are well described. There is a particularly illuminating review of the arguments for unilateral or bilateral representation of the macula in the discussion on macular sparing.

This book will prove invaluable for ophthalmologists in training, field technicians, orthoptists and ophthalmic nurses. It also provides an excellent refresher course for established ophthalmologists and there is a selfstudy multiple choice test at the end to test memory faculties.

N.J.C. Sarkies

\section{ERRATA}

Eye Volume 11 part 5, pp. 759-760 Letter to the Journal: Ocular exposure to $\mathrm{CS}$ gas

The authors of the letter are Dr A. W. Kiel and Mr A. J. Luff. We apologise to Mr Luff for the inadvertent omission of his name.

Eye Volume 12 part 1, pp. 154-156 Letter to the Journal: An uncommon presentation of ocular ischaemia syndrome The correct order of authors for this letter is Mr David Broadway, Dr Christina Flaxel and Mr Zdenek Gregor. 